\title{
Apatinib combined with PD-L1 blockade synergistically enhances antitumor immune responses and promotes HEV formation in gastric cancer
}

\section{Yu Zhang}

Fudan University Shanghai Cancer Center

\section{Fei Wang}

Fudan University Shanghai Cancer Center

Hao-ran Sun

Huashan Hospital Fudan University

\section{Ya-kai Huang}

Fudan University Shanghai Cancer Center Jian-peng Gao

Fudan University Shanghai Cancer Center Hua Huang ( $\square$ huahuang@fudan.edu.cn ) Fudan University Shanghai Cancer Center https://orcid.org/0000-0002-8936-3371

\section{Research Article}

Keywords: gastric cancer, apatinib, PD-L1 blockade, HEV

Posted Date: March 19th, 2021

DOI: https://doi.org/10.21203/rs.3.rs-95102/v2

License: (c) (i) This work is licensed under a Creative Commons Attribution 4.0 International License. Read Full License

Version of Record: A version of this preprint was published at Journal of Cancer Research and Clinical Oncology on April 23rd, 2021. See the published version at https://doi.org/10.1007/s00432-021-03633-3. 


\section{Abstract \\ Purpose}

Apatinib, an antiangiogenic drug, has showed beneficial effects only in a fraction of advanced gastric cancer (GC) patients. Given the recent success of immunotherapies, combination of apatinib with immune checkpoint inhibitor may provide sustained and potent antitumor responses.

\section{Methods}

Immunocompetent mice with subcutaneous MFC tumors grown were given combination of apatinib and anti-PD-L1 antibody therapy. GC tissues from patients undergoing curative resection in China were collected, and the density of HEVs, MSI status and tumor-infiltrated lymphocytes were analyzed by immunohistochemical staining.

\section{Results}

Combined apatinib and PD-L1 blockade therapy synergistically delayed tumor growth and increased survival in MFC-bearing immunocompetent mice. The combination therapy promoted antitumor immunity by increasing the ratio of $\mathrm{CD}^{+}$cytotoxic $\mathrm{T}$ cells to Foxp3 ${ }^{+}$Treg cells, the accumulation of $\mathrm{CD} 20^{+} \mathrm{B}$ cells and the Th1/Th2 cytokine ratio (IFN-y/IL-10). The combination therapy induced the formation of HEVs through activation of LT $\beta R$ signaling, thus promoting $C D 8^{+}$cytotoxic $T$ cell and $C D 20^{+}$ B cell infiltration in tumors. In clinical GC samples, the density of HEVs positively correlated with the intratumoral infiltration of $\mathrm{CD} 8^{+}$cytotoxic $\mathrm{T}$ cells and $\mathrm{CD} 2 \mathrm{O}^{+} \mathrm{B}$ cells. MSI-high GC showed a higher density of $\mathrm{HEVs}, \mathrm{CD} 8^{+}$cytotoxic $\mathrm{T}$ cells and $\mathrm{CD} 20^{+} \mathrm{B}$ cells than MSS/MSI-low GC. GC patients with high densities of $\mathrm{HEVs}, \mathrm{CD} 8^{+}$cytotoxic $\mathrm{T}$ cells and $\mathrm{CD} 2 \mathrm{O}^{+} \mathrm{B}$ cells had an improved prognosis with superior overall survival.

\section{Conclusion}

Combining apatinib with PD-L1 blockade treatment synergistically enhances antitumor immune responses and promotes HEV formation in GC.

\section{Introduction}

Gastric cancer (GC) is a lethal malignancy that continues to have a major impact on global health (Bray et al. 2018). Despite improvements in diagnostic techniques and progress in surgical and nonsurgical therapies, the prognosis of GC remains poor (Russo and Strong 2019). The immune system is a critical and complex regulator of tumor evolution. Tumor angiogenesis not only supplies nutrients and oxygen to 
cancer tissues but also actively modulates antitumor immune activity (Kandalaft et al. 2011). The angiogenic vasculature of the tumor provides a barrier to prevent effective leukocytes priming for tumor control, which presents a significant challenge to the success of immunotherapy (Ramjiawan et al. 2017). VEGF and its receptor, VEGFR-2, play a central role in tumor angiogenesis (Jayson et al. 2016). It has been reported that inhibition of the VEGFR-2 pathway has the capability to increase the trafficking of T cells into tumors and decrease the number and function of immune cells with inhibitory phenotypes (Huang et al. 2012). Apatinib, a functional inhibitor of VEGF/VEGFR-2, has recently been approved and indicated for advanced gastric cancer after the failure of two or more lines of systemic therapy in China (Geng et al. 2018). On this basis, inhibiting VEGF/VEGFR-2 signaling with apatinib may induce a transient state of vessel remodeling to facilitate lymphocyte infiltration in tumor tissues.

PD-1/PD-L1 immune checkpoint blockade therapy has afforded patients with malignancies the potential for long-term survival. Unfortunately, only a minority of the total treated patients respond to immune checkpoint blockade (Gibney et al. 2016). The presence of tumor-infiltrating lymphocytes (TILs) has been reported to have a dominant role in the response to immunotherapy (Fridman et al. 2017). Since crosstalk between the tumor immune microenvironment and the tumor vasculature contributes to tumor immune evasion, combining therapeutic regimens targeting vascular factors with immune checkpoint blockade may provide deep and durable antitumor immune responses.

High endothelial venules (HEVs) are anatomically distinct postcapillary venules found in lymphoid tissues and facilitate lymphocyte trafficking into secondary lymphoid organs (Girard et al. 2012). In cancer, HEVs can arise spontaneously and are often associated with favorable clinical outcomes (Pfuderer et al. 2019; Martinet et al. 2011; Wirsing et al. 2018; Avram et al. 2013; Hill et al. 2018). Although preliminary evidence suggests an association between tumor-associated HEVs and clinical outcomes in several solid tumors, the effect of tumor-associated HEVs and their association with lymphocyte infiltration, immune orientation, response to targeted therapy and clinical outcome in GC are still not completely understood.

In the present study, we investigated the feasibility of combining apatinib with PD-L1 blockade immunotherapy as a therapeutic option in gastric cancer and whether synergism between apatinib and PD-L1 blockade involves facilitating lymphocyte infiltration through HEV formation.

\section{Materials And Methods}

\section{Patients and Specimens}

Tumor tissue samples were obtained from patients with gastric adenocarcinoma who underwent curative resection at the authors' institutions. All patients were diagnosed with gastric adenocarcinoma and had yet to receive any preoperative cancer treatment. Patients with infectious diseases, autoimmune disease or multi-primary cancers were excluded. The histopathologic diagnosis was based on the World Health Organization criteria. The clinical stages of tumors were determined according to the tumor, node, metastases (TNM) classification system of the International Union Against Cancer (8th edition). For 
immunohistochemical and prognostic analysis, tumor tissues from 192 gastric adenocarcinoma patients undergoing curative resection at the authors' institutions between 2011 and 2012 were randomly collected. Overall survival was defined as the interval between surgery and death or between surgery and the last observation point. For surviving patients, the data were censored at the last follow-up evaluation. For analyzing the relation between HEV, DCs and MSI status, tumor tissues from 33 gastric adenocarcinoma patients undergoing curative resection at the authors' institutions in 2019 were randomly collected. For analyzing the relation between HEV and DCs, tumor tissues from 42 gastric adenocarcinoma patients undergoing curative resection at the authors' institutions in 2020 were randomly collected. For analyzing the relation between HEV and lymphocytes migration related genes, tumor tissues from 18 gastric adenocarcinoma patients undergoing curative resection at the authors' institutions in 2020 were randomly collected. Clinicopathological characteristics of these patients are presented in Supplementary Table 1-4. Clinical samples were collected from patients after obtaining informed consent, and all related procedures were performed with the approval of the internal review and ethics boards of the authors' institutions.

\section{Reagents and antibodies}

Apatinib was obtained from Hengrui Medicine Co. Ltd. (Jiangsu, China). PD-L1-blocking antibody (clone 10F.9G2) was obtained from BioXcell (West Lebanon, NH, USA). LT $\beta R$ antagonist was obtained from TuBio (Cat\# 71122). Recombinant human and mouse IFN-y protein was obtained from R\&D Systems(Cat\#285-IF; Cat\#485-MI).

\section{Western blot analysis}

The cells were lysed with RIPA Lysis Buffer (Santa Cruz Biotechnology, CA, USA) containing protease inhibitor (Roche Corp., Basal, Swiss) and phosphatase inhibitor (Roche Corp., Basal, Swiss). The protein concentration was determined using a BCA Protein Assay Kit (Beyotime Biotechnology, Shanghai, China) and equalized before loading. A total of 25 to $50 \mathrm{mg}$ of proteins were separated by SDS-PAGE and transferred onto polyvinylidene difluoride membranes. Membranes were blocked and blotted with the relevant antibodies. Horseradish peroxidase-conjugated secondary antibodies were detected with an enhanced chemiluminescence reagent (Millipore Corp., MA, USA). All information of primary antibodies used for western blot and immunohistochemical analysis were shown on Supplementary Table 5. All secondary antibodies were purchased from Jackson ImmunoResearch (Philadelphia, PA, USA).

\section{RNA Isolation, Reverse-Transcription, and Quantitative Real-time PolymeraseChain Reaction (qRT-PCR)}

Total RNA was extracted from cell lines and tumor tissues using Trizol reagent (Invitrogen, Carlsbad, CA), and reverse-transcribed into single-stranded cDNA using PrimeScript ${ }^{\mathrm{TM}}$ RT Reagent Kit (TaKaRa Biotechnology) in accordance with the manufacturer's instructions. Quantitative real time polymerase chain reaction (qRT-PCR) was performed with SYBR Green qPCR Master Mix (DBI Bioscience) in accordance with the manufacturer's instructions. qRT-PCR specific primer sequences were showed in 
Supplementary Table 6. GAPDH was used as an internal control. Relative mRNA levels were calculated based on the $\mathrm{Ct}$ values to yield a $2^{-\triangle \Delta \mathrm{Ct}}$ value for relative expression of each transcript.

\section{Statistical analysis}

Statistical analyses were performed using SPSS 15.0 for Windows (SPSS, Inc., Chicago, IL). Quantitative data between groups were compared using one-way ANOVA, and Student's $t$ test was used to compare data between two groups. Categorical data were analyzed using chi-square test or Fisher exact test. OS and cumulative recurrence rates were calculated by the Kaplan-Meier method, and differences were analyzed using the log-rank test. Univariate and multivariate analyses were performed using the Cox proportional hazards regression model. $P<0.05$ was considered statistically significant.

Detailed descriptions of the Materials and Methods are available in the Supplementary Materials and Methods.

\section{Results}

\section{Apatinib combined with PD-L1 blockade synergistically impedes tumor growth and improves OS in a GC mouse model}

To investigate whether combined therapy would be more efficacious than apatinib or PD-L1 blockade monotherapy, we performed a therapeutic study in a cell line-derived xenograft (CDX) murine model. The mouse GC cell line MFC was inoculated subcutaneously into the flanks of immunocompetent mice. Once tumors were palpable, mice were randomized and systemically treated with isotype control antibody, apatinib, anti-PD-L1 antibody or the combination of anti-PD-L1 antibody and apatinib until the study endpoint. The therapy protocol is shown in Fig. 1a. The groups treated with apatinib monotherapy and anti-PD-L1 antibody monotherapy had significantly improved median overall survival (OS) compared with the control group (Fig. 1b). Strikingly, the combined treatment of apatinib and anti-PD-L1 antibody led to a substantial improvement in median OS compared with control or a single agent alone (Fig. 1b). To further elucidate whether the combination of apatinib and anti-PD-L1 antibody exerted synergistic treatment effects, we calculated the predicted additive OS curve according to the method in a published paper (Meder et al. 2018) and compared the predicted additive OS curve with the observed OS curve of the combined apatinib and anti-PD-L1 antibody therapy group. We found that the observed OS (median survival not detected until 70 days) of the MFC-bearing mice treated with combined apatinib and anti-PDL1 antibody therapy was significantly longer than the predicted additive OS (median survival=54 days) upon the combination therapy $(P=0.013)$, which indicated synergism between apatinib and PD-L1 blockade (Fig. 1C). Moreover, after 2 weeks of treatment, we found that treatment with apatinib or anti-PDL1 antibody alone significantly inhibited the growth of MFC tumors in immunocompetent mice, and combination treatment with apatinib and anti-PD-L1 antibody led to a further reduction in tumor volume and tumor weight (Fig. 1d,1e). In addition, combined treatment dramatically decreased the number of Ki67-positive proliferative tumor cells compared with the control or a single agent alone (Fig. 1f). 
Collectively, these data suggest that treatment of MFC-bearing mice with combined apatinib and PD-L1 blockade therapy synergistically improves OS and inhibits tumor growth compared with apatinib or PD-L1 blockade monotherapy.

\section{Combined apatinib and PD-L1 blockade reprograms the immune microenvironment and promotes antitumor immunity in a GC mouse model}

We next examined how apatinib monotherapy, anti-PD-L1 monotherapy and combined apatinib and PDL1 therapy impact lymphocyte infiltration in MFC tumors. Immunohistochemical analysis showed that apatinib treatment alone increased the number of $\mathrm{Foxp}^{+}{ }^{+}$Treg cells, while the number of Foxp $3^{+}$Treg cells was not significantly altered upon anti-PD-L1 monotherapy and combined apatinib/anti-PD-L1 therapy compared with control treatment (Fig. 2a). Using immunofluorescence staining (IF), we found that in control MFC tumors Foxp3 ${ }^{+}$cells were mainly PD-1-negative cells, while apatinib treatment significantly increased the number of PD- $1^{+}$Foxp3 ${ }^{+}$Treg cells, and blocking PD-1/PD-L1 axis by anti-PD$\mathrm{L} 1$ antibody could abrogate the increased tumor infiltration by PD $-1^{+}$Foxp $3^{+}$Treg cells mediated by apatinib (Supplementary Fig.1). Moreover, the number of $\mathrm{CD} 8^{+} \mathrm{T}$ cells and $\mathrm{CD} 20^{+} \mathrm{B}$ cells was significantly increased upon anti-PD-L1 and combined apatinib/anti-PD-L1 therapy but was not significantly altered upon apatinib therapy compared with those in the control group (Fig. 2b,2c). Furthermore, the combination therapy led to a higher ratio of $\mathrm{CD} 8^{+} \mathrm{T}$ cells to Foxp $3^{+}$Treg cells, indicating a shift towards immune activation rather than an immunosuppressive microenvironment (Fig. 2d). To validate the activation of tumor-infiltrating $\mathrm{CD} 8^{+} \mathrm{T}$ cells after PD-L1 blockade, we evaluated biomarkers (IFN-y, TNFa and IL-2) indicative of $\mathrm{CD}^{+}{ }^{+} \mathrm{T}$ cell activation in mouse plasma at the study endpoint after treatment. Significantly increased levels of IFN- - , TNFa and IL-2 were observed when mice were treated with the apatinib/anti-PD-L1 combination treatment, while apatinib or anti-PD-L1 monotherapy only weakly increased activated $C D 8^{+} T$ cells (Fig. 2e). Furthermore, when evaluating the Th1/Th2 cytokine balance by ELISA, we found that the ratio of Th1 to Th2 cytokines (IFN-y divided by IL-10) was significantly increased upon combined apatinib and ani-PD-L1 therapy compared with each agent alone (Fig. 2f). Collectively, these data suggest that combined apatinib and PD-L1 blockade reprograms the immune microenvironment and promotes antitumor immunity in a GC mouse model.

\section{Apatinib increases PD-L1 expression in gastric cancer cells via hypoxia and IFN-y expression}

We next investigated the impact of apatinib monotherapy, anti-PD-L1 monotherapy and combined apatinib/anti-PD-L1 treatment on PD-L1 expression in GC tumors. Immunohistochemical analysis showed that the expression of PD-L1 was dramatically increased in tumor samples upon apatinib monotherapy and combined apatinib/anti-PD-L1 therapy compared with anti-PD-L1 monotherapy (Fig. 3a). We further examined whether apatinib exerted a direct effect on PD-L1 expression in GC cells. We found that apatinib did not result in an appreciable change in PD-L1 expression in GC cells under hypoxic and normoxic conditions (Supplementary Fig.2a). Hypoxia, which can be caused by antiangiogenic therapy, has been reported to increase the expression of PD-L1 on tumor cells via HIF-1a (Noman et al. 
2014). Therefore, we investigated whether apatinib-induced hypoxia increases PD-L1 expression in MFC tumors. We observed increased nuclear expression of HIF-1a in apatinib- and combined apatinib/anti-PD-

L1-treated MFC-bearing mouse models compared with that in control-treated mice, accompanied by increased PD-L1 expression (Fig. 3b). We also observed that during apatinib treatment, PD-L1 was strongly correlated with HIF-1a expression (Fig. 3c), indicating PD-L1 expression by GC cells, particularly in hypoxic regions. Given that IFN-y has been reported to mediate PD-L1 expression in tumor cells (Lee et al. 2006), we next investigated whether IFN- $\gamma$ was involved in the PD-L1 expression induced by apatinib treatment in GC cells. As shown in Fig. 3d,3e, IFN- $\gamma$ dramatically induced PD-L1 expression in GC cells. By ELISA, we found that IFN- $\gamma$ was significantly increased in the plasma of MFC-bearing mice upon apatinib and apatinib/anti-PD-L1 combination treatment (Fig. 2e). The expression of IFN- $\gamma$-mediated genes, CXCL10, MX1 and IFIT3 (Samarajiwa et al. 2009), was also higher in MFC cells isolated from tumors treated with apatinib and apatinib/anti-PD-L1 combined therapy compared with that in the control or antiPD-L1 group (Fig. 3f). These results suggest that apatinib enhances the expression of PD-L1 partially by inducing IFN- $\gamma$ expression as well as by inducing a hypoxic microenvironment in GC.

\section{Combined apatinib and PD-L1 blockade induces HEV formation that is associated with LT $\beta R$ signaling activation}

Reprogramming the tumor vasculature by normalization could lead to a shift towards an immuneactivated microenvironment (Huang et al. 2012); therefore, we hypothesized that combined apatinib and PD-L1 blockade therapy may affect the tumor vasculature in GC. Immunohistochemical analysis showed that the vessel density, as indicated by the number of CD-31-positive vessels per area, was dramatically reduced in tumor samples upon apatinib monotherapy and combined apatinib/anti-PD-L1 therapy but not in anti-PD-L1 monotherapy-treated tumors (Fig. 4a). HEVs, which are structurally distinct blood vessels, are thought to facilitate the generation of tissue-destroying lymphocytes inside chronically inflamed tissues and cancers (Girard et al. 2012). Using immunohistochemical staining for the HEV-specific marker MECA-79 (Rosen 2004), we found that naive MFC tumors were devoid of MECA-79+ vessels, whereas MECA-79+ vessels were markedly induced by combined apatinib/anti-PD-L1 therapy (Fig. 4b). Previous studies suggested that continuous stimulation of lymphotoxin- $\beta$ receptor (LT $\beta R$ ) on HEV endothelial cells by $C D 11 c^{+} D C s$ is essential for the induction and maintenance of HEVs in lymphoid tissues (Moussion and Girard 2011; Martinet et al. 2013; Wculek et al. 2020). Indeed, the number of CD11 ${ }^{+}$DCs was significantly increased in MFC tumor tissues upon combined apatinib/anti-PD-L1 treatment (Fig. 4c). We next examined the role of LT $\beta R$ signaling in HEV formation during combined apatinib/anti-PD-L1 treatment. We found that an LT $\beta R$ antagonist exerted little effect on the proliferation of GC cell lines in vitro (Supplementary Fig. 2b). We next tested the effect of combined treatment with an LT $\beta R$ signaling antagonistic antibody and combined apatinib/anti-PD-L1 therapy in an immunocompetent mouse GC model. As shown in Fig. 4d-f, no significant tumor suppression was observed in the LT $\beta R$ antagonist-onlytreated group compared with the control group. Meanwhile, combined apatinib/anti-PD-L1 treatment significantly decreased the tumor burden of MFC tumors by approximately $80 \%$. Notably, the addition of an LT $\beta R$ antagonist during combined apatinib/anti-PD-L1 treatment substantially weakened the 
antitumor effect (Fig. 4d-f). Immunohistochemistry was used to evaluate the density of MECA-79 ${ }^{+} \mathrm{HEVs}$, $\mathrm{CD} 8^{+} \mathrm{T}$ cells and $\mathrm{CD} 20^{+} \mathrm{B}$ cells in the xenograft tumors. We found that the HEV formation, and the CD $8^{+}$ $\mathrm{T}$ cell and $\mathrm{CD} 2 \mathrm{O}^{+} \mathrm{B}$ cell infiltration driven by combined apatinib/anti-PD-L1 treatment were also blocked by treatment with an LT $\beta R$ antagonist (Fig. 4g,4h). These data support the idea that LT $\beta R$ signaling activated by DCs contributes to HEV formation during combined apatinib/anti-PD-L1 treatment.

\section{Presence of HEVs correlates with clinical outcome in GC patients}

To identify the presence of HEVs in human GC, tumor tissues from 192 surgically resected gastric adenocarcinoma patients were analyzed for expression of the HEV-specific marker MECA-79. Vessels expressing MECA-79 were detected in the majority of GC tumors analyzed (118/192). A portion of MECA79-positive vessels displayed a cuboidal endothelial appearance, typical of lymph node HEVs (Fig. 5a). MECA-79 was also expressed on endothelial cells with a flat morphology, typical of the tumor vasculature (Fig. 5a). The density of MECA-79-positive HEVs varied in patients, ranging from 0 to $6.2 \mathrm{HEVs}$ per $\mathrm{mm}^{3}$ (median: $0.8 \mathrm{HEVs} / \mathrm{mm}^{3}$, mean: $1.1 \mathrm{HEVs} / \mathrm{mm}^{3}$ ) (Fig. 5a). Moreover, immunostaining of the pan-vascular endothelial cell markers CD31 and MECA-79 in GC samples demonstrated that the percentage of MECA$79^{+}$vessels ranged from $1 \%$ to $11 \%$ of the $\mathrm{CD} 31^{+}$vessels (Fig. $5 \mathrm{~b}$ ), and no significant correlation between intratumoral MECA- $79^{+}$and $\mathrm{CD} 31^{+}$vessels was observed (Fig. 5c), suggesting that the differences in angiogenesis were not responsible for the differences in the density of HEVs in gastric tumors. Furthermore, a strong association between $\mathrm{DC}$-LAMP ${ }^{+}\left(\mathrm{LAMP}^{+}\right)$mature dendritic cells and the presence of MECA79 ${ }^{+}$high endothelial venules was identified (Fig. 5d). We further tested the correlations between the density of MECA-79-positive HEVs and the clinicopathologic status and prognosis of patients with GC. We found that the density of HEVs in gastric adenocarcinoma samples of early stage (TNM stage I$I I, n=81$ ) was significantly elevated compared to that in gastric adenocarcinoma samples of advanced stage (TNM stage III-IV, $n=111$ ) (Fig. 5e; Supplementary Table 7). Further analyses showed that a low density of HEVs in gastric adenocarcinoma was positively associated with relatively higher tumor size, vascular invasion and lymph node metastasis than a high density of HEVs (Fig.5f-h; Supplementary Table 7). Kaplan-Meier analysis revealed that a low density of HEVs predicted a poor prognosis for overall survival in gastric adenocarcinoma patients after surgery (Fig. 5i). Cox regression multivariate analysis also revealed that HEV density, tumor differentiation, lymph node metastasis and TNM stage were independent prognostic factors for the survival of gastric adenocarcinoma patients (Fig. 5j; Supplementary Table 8).

\section{HEVs predict lymphocyte infiltration into the tumor in GC patients}

Because HEVs are involved in the large-scale migration of lymphocytes from the blood into lymph nodes, we next investigated the relationship between HEVs and immune infiltration in GC. Subsequently, we quantified the number of tumor-infiltrating $\mathrm{CD}^{+} \mathrm{T}$ cells, $\mathrm{CD} 8^{+} \mathrm{T}$ cells and $\mathrm{CD} 2 \mathrm{O}^{+} \mathrm{B}$ cells by $\mathrm{IHC}$ staining in resected tumors from 192 gastric adenocarcinoma patients. We found infiltration of $\mathrm{CD}^{2} \mathrm{O}^{+} \mathrm{B}$ cells in $39 \%$ of patients; $27 \%$ of the tumors had $\mathrm{CD} 20^{+} \mathrm{B}$ cell localized in clusters, and $34 \%$ were devoid of $\mathrm{CD} 20^{+} \mathrm{B}$ 
cells (Fig. 6a). Notably, CD20 ${ }^{+}$B cell clusters were in all cases colocalized with MECA79 ${ }^{+}$high endothelial venules, as well as $\mathrm{CD}^{+}$and $\mathrm{CD} 8^{+} \mathrm{T}$ cells, which indicates the formation of tertiary lymphoid structures (TLSs) (Fig. 6a). In contrast, the infiltration of $\mathrm{CD}^{+}{ }^{+}$T cells was found in $49 \%$ of cases, and $27 \%$ of tumors had $\mathrm{CD}^{+} \mathrm{T}$ cells localized outside of such TLSs (Fig. 6a). Furthermore, we observed a strong correlation between the number of HEVs and intratumoral CD8 ${ }^{+} T$ cells $(r=0.669 ; P<0.001)$ and $C D 20^{+} B$ cells $(r=0.751 ; P<0.001)$ (Fig. 6b,6c). The mRNA expression levels of genes associated with naive and central memory $\mathrm{T}$ and B lymphocyte migration, including CCL 19, CCL21, CXCL 13 and CCR7 (von Andrian and Mempel 2003), were significantly elevated in the HEV-high versus HEV-low tumors (Fig. 6d).

Microsatellite instability (MSI) is a tumor molecular phenotype caused by a defect in the DNA mismatch repair (MMR) system machinery, and MSI-H-induced immunogenic neoantigens are thought to trigger a potent antitumor response in the presence of immune checkpoint blockade (Le DT et al. 2017). We therefore explored the relationship between MSI and the immune phenotype in the GC microenvironment and whether HEVs were correlated with immune infiltration in MSI GCs. By comparing $21 \mathrm{MSI}(\mathrm{MSI}-\mathrm{H}=10$, MSI-L=11) against 12 MSS gastric adenocarcinoma tissue samples, we observed that the abundance of intratumoral $\mathrm{CD}^{+} \mathrm{T}$ cells and $\mathrm{CD} 20^{+} \mathrm{B}$ cells in MSI-H gastric adenocarcinoma tissues was higher than that in the MSI-L or MSS GC samples (Fig. 6e). Strikingly, the numbers of $C D 8^{+} T$ cells and $C D 20^{+} B$ cells in MSI-L tumors were comparable to those in MSS samples (Fig. 6e). Nest, we tested whether HEVs were correlated with the abundant immune infiltration in MSI GCs. As shown in Fig. 6f, the density of HEVs was higher in MSI-H than MSI-L or MSS GC samples. However, the density of HEVs was comparable between MSI-L and MSS GC samples. Moreover, the DC-LAMP ${ }^{+}\left(\mathrm{LAMP3}^{+}\right)$DCs in MSI-H gastric adenocarcinoma tissues was higher than that in the MSI-L or MSS GC samples, and the numbers of DCLAMP $^{+}\left(\right.$LAMP3 $^{+}$) DCs in MSI-L tumors were comparable to those in MSS samples (Supplementary Fig.3a). We further analyzed the expression of LT $\beta R$ and its ligands LTa, LT $\beta$ and LIGHT by qRT-PCR in 10 $\mathrm{MSI}(\mathrm{MSI}-\mathrm{H}=5, \mathrm{MSI}-\mathrm{L}=5)$ and $5 \mathrm{MSS}$ gastric adenocarcinoma tissue samples. We found that the expression of the LT $\beta R, L T \beta$ and LIGHT, but not LTa, in the MSI-H samples was significantly higher than that in MSI-L or MSS GC samples, whereas no significant difference in the expression of LT $\beta R, L T a, L T \beta$ and LIGHT was observed between MSI-L and MSS GC samples (Supplementary Fig.3b). These suggested that activation of LT $\beta R$ signaling by DCs may contribute to the increased tumor-associated HEVs formation in MSI-H GCs.

Survival analysis revealed that the presence of TLSs was associated with improved patient outcome (Fig. $6 \mathrm{~g})$. Gastric adenocarcinoma patients in the $\mathrm{CD} 8^{+} \mathrm{T}$ cell-high or $\mathrm{CD} 2 \mathrm{O}^{+} \mathrm{B}$ cell-high group exhibited significantly longer OS than those in the CD8+ T cell-low or CD20+ B cell-low group (Fig. 6h,6i), and when $\mathrm{HEV}$ s, $\mathrm{CD} 8^{+} \mathrm{T}$ cells and $\mathrm{CD} 20^{+} \mathrm{B}$ cells were considered together, we observed that patients with low levels of all HEVs, $C D 8^{+} T$ cells and $C D 20^{+} B$ cells exhibited the shortest $O S$, whereas those with high levels of all HEVs, CD8 ${ }^{+} \mathrm{T}$ cells and $\mathrm{CD} 20^{+} \mathrm{B}$ cells displayed the best prognosis (Fig. 6j).

\section{Discussion}


Antiangiogenic therapy has been validated as an important anticancer treatment modality through clinical trials, which has added VEGF pathway-targeting drugs into the therapeutic armory (Jayson et al. 2016). Apatinib is an orally administered VEGFR-2-targeting drug that has demonstrated improved OS and PFS in patients with advanced-stage GC refractory to two or more lines of prior chemotherapy (Geng et al. 2018). However, responses are typically transient, and most responsive patients develop resistance over time. Given the recent success of immunotherapies, combinations of antiangiogenic drugs with immune checkpoint inhibitors may provide sustained and potent antitumor responses. Several preclinical studies have assumed that the potential of combined antiangiogenic therapy with immune checkpoint inhibitors for enhancing antitumor immunity in cancer patients stems from different mechanisms, mainly converging on reprogramming of the vasculature and evasion of the immunosuppressive tumor microenvironment (Huang et al. 2012; Allen et al. 2017; Schmittnaegel et al. 2017; Shigeta et al. 2020). In this study, we described that combining apatinib, a VEGFR-2 TKI, with PD-L1 blockade synergistically improves treatment efficacy in GC. The combination therapy not only directly counteracted the apatinib monotherapy-induced PD-L1 upregulation caused by hypoxia and IFN- $\gamma$ but also induced the formation of HEVs, which promoted antitumor immunity by recruiting activated lymphocytes to infiltrate the tumor site, thus producing successful eradication of GC cells.

The clinical development of checkpoint inhibitor-based immunotherapy has revolutionized cancer therapy. Despite these advances, in some cases, improvements in PFS and OS are modest. Mechanisms of resistance to checkpoint inhibitors are probably multifactorial and can include an absence of PD-L1, low levels of intratumor immunological activity, or both (Pitt et al. 2016). Antiangiogenic therapy with sorafenib or sunitinib has been reported to increase PD-L1 expression in solid tumors and PD-1 expression on T cells, including renal cell carcinoma and hepatocellular carcinoma (Ramjiawan et al. 2017; Chen et al. 2015; Liu et al. 2015). In line with a previous study, we found that apatinib treatment could promote the expression of PD-L1 in GC cells and PD-1 expression in Foxp3 ${ }^{+}$Treg cells. It has been well appreciated that PD-L1 expression can be induced by hypoxia via the hypoxia-inducible factor HIF1-a and by IFN-ץ (Noman et al. 2014; Lee et al. 2006). Shigeta et al. found that PD-L1 expression was induced in a paracrine manner upon anti-VEGFR-2 blockade in endothelial cells in part through IFN-y expression by HCC cells (Shigeta et al. 2020). However, Allen et al. have shown that PD-L1 expression is increased by IFN-y-producing T lymphocytes during VEGF/VEGFR blockade treatment (Allen et al. 2017). Our present data show that PD-L1 expression by GC cells was particularly high in hypoxic regions and that PD-L1 was strongly correlated with HIF-1 a expression during apatinib treatment. Additionally, IFN-y expression in the plasma of GC-bearing mice was significantly increased upon apatinib treatment, accompanied by the substantially increased expression of IFN- $\gamma$-inducible genes in GC cells, and IFN-y could significantly upregulate PD-L1 expression in GC cells in vitro. Emerging evidences suggest that the activation of PD1/PD-L1 pathway renders the expansion, maintenance and function of PD- $1^{+}$Foxp $3^{+}$Treg cells within tumor sites, and anti-angiogenic treatment can increase the PD-1 expression on T cells. We found that apatinib treatment significantly increased the number of PD- $1^{+} F o x p 3^{+}$cells, which could interact with the upregulated PD-L1 on tumor cells, subsequently leading to the increased intratumoral infiltration of PD$1^{+} \mathrm{Foxp} 3^{+}$cells. Blocking PD-1/PD-L1 axis by anti-PD-L1 antibody could abrogate the increased tumor 
infiltration by Foxp $3^{+}$cells most of which are PD- $1^{+}$Foxp3+ subsets mediated by apatinib. Therefore, PD1/PD-L1 blockade by anti-PD-L1 antibody in combination with aptinib did not prevent PD-L1 upregulation due to hypoxia and IFN-y induced by apatinib, but it blocked PD-1/PD-L1 interaction between tumor cells and immune cells and then increased the intratumoral accumulation of $\mathrm{CD} 8^{+} \mathrm{T}$ cells and $\mathrm{CD} 2 \mathrm{O}^{+} \mathrm{B}$ cells and the ratio of $\mathrm{CD} 8^{+}$cytotoxic $T$ cells to Foxp $3^{+}$Treg cells, which led the tumor growth inhibiton. These indicated that though anti-PD-L1 antibody can't prevented the upregulation of PD-L1 which is resulted from the increased expression of HIF-1a and IFN- $\gamma$ caused by apatinib, it can block the PD-L1/PD-1 interaction mediated suppression of immune cell responses, which led to the consequent tumor growth suppression.

Lymphocyte trafficking has a crucial role in antitumor immunity because it maximizes the probability that lymphocytes will encounter tumor antigens. Under homeostatic conditions, naive lymphocytes continuously circulate from the blood to lymphoid organs by crossing the walls of HEVs (Girard et al. 2012). Recent studies have demonstrated that, similar to their counterparts localized in lymphoid organs, tumor-associated HEVs are frequently observed in the stroma of human solid tumors and represent major gateways controlling leukocyte entry into compartmentalized regions of tumor tissues. This scenario is supported by studies showing that high densities of tumor-associated HEVs correlate with elevated levels of TILs and/or with favorable clinical outcome in solid tumors, including melanoma, colorectal cancer, oral squamous cell carcinoma and breast cancer (Pfuderer et al. 2019; Martinet et al. 2011; Wirsing et al. 2018; Avram et al. 2013). A previous study reported the presence of HEVs in GC (Hill et al. 2018), but their association with lymphocyte infiltration and clinical outcome in GC patients were largely unknown. In the current study, we found that GC tissues contained MECA-79+ HEVs with phenotypic characteristics similar to those of HEVs in lymphoid organs. The density of MECA- $79^{+}$tumor HEVs was strongly reduced during GC development from the early stage to the late stage, suggesting that the loss of tumor-associated HEVs is a critical step during GC progression. The density of HEVs was also strongly correlated with the density of tumor-infiltrating $\mathrm{CD} 8^{+} \mathrm{T}$ cells and $\mathrm{CD} 20^{+} \mathrm{B}$ cells and $\mathrm{OS}$ in primary gastric adenocarcinoma patients. Both $\mathrm{CD} 8^{+} \mathrm{T}$ cells and $\mathrm{CD} 20^{+} \mathrm{B}$ have the ability to inhibit tumor progression and exert favorable impacts on the clinical outcomes of numerous cancers (Mahmoud et al. 2011; Sato et al. 2020). In line with a previous study, we found that the presence of $\mathrm{CD} 8^{+} \mathrm{T}$ cells and $\mathrm{CD} 20^{+} \mathrm{B}$ cells surrounding HEVs was associated with improved OS in gastric adenocarcinoma patients. These findings indicate that tumorassociated HEVs are related to clinical outcomes by supporting a constant influx of $\mathrm{CD}^{+} \mathrm{T}$ cells and CD20 ${ }^{+} \mathrm{B}$ cells in the tumor stroma. Tertiary lymphoid structures (TLSs) have been identified in a wide range of cancers, including GC, but their presence is highly variable between cancer types as well as between patients (Sautes-Fridman et al. 2019). Tumor-associated TLSs are not only surrogate markers of a rapid immune response; instead, they are also thought to be associated with improved prognosis and clinical response to immune checkpoint inhibitors (Helmink et al. 2020; Petitprez et al. 2020; Cabrita et al. 2020). Our study confirmed that TLSs are associated with favorable prognosis in GC patients. The role of TLSs in the prediction of response to immune therapy in GC still needs to be explored in the future. Microsatellite instability (MSI), arising from deficient DNA mismatch repair (dMMR), commonly presents 
as an increased mutation rate and dense lymphocyte infiltration (Cortes-Ciriano et al. 2017). As a result, assessment of either dMMR by immunohistochemistry or MSI is recommended for several solid cancers to guide treatment decisions (ESO et al. 2020). Whether improved lymphocyte recruitment by tumorassociated HEVs contributes to the pronounced immune infiltration in MSI GCs is incompletely understood. Bento et al. found no striking differences in $\mathrm{HEV}$ density between $\mathrm{MSI}^{+}$and $\mathrm{MSI}^{-} \mathrm{CRC}$ tumors by analyzing only four MSI CRC samples (Bento et al. 2015). However, Pfuderer et al. showed elevated HEV densities in MSI CRCs compared with MSS CRCs in a larger sample size of $\mathrm{MSI}^{+} \mathrm{CRC}$ samples (Pfuderer et al. 2019). This study is, to our knowledge, the first to uncover a markedly higher tumorassociated HEV density in MSI GC tumors than MSS GC tumors, and this increased HEV density was accompanied by increased infiltration of $\mathrm{CD} 8^{+} \mathrm{T}$ cells and $\mathrm{CD} 2 \mathrm{O}^{+} \mathrm{B}$ cells in the tumor tissues. Thus, our current finding underscores the significant contribution of tumor-associated HEVs in immune responses against $\mathrm{MSI}^{+} \mathrm{GC}$.

Emerging evidence suggests that the nonactivated endothelium of tumor tissues can rapidly transform into HEV-like vessels in response to certain targeted drugs. Allen and colleagues reported that treatment with a combination of anti-VEGFR2 and anti-PD-L1 antibodies induced HEVs to promote lymphocyte infiltration in PyMT (polyoma middle T oncoprotein) breast cancer and RT2-PNET (Rip1-Tag2 pancreatic neuroendocrine tumors) but not in glioblastoma (GBM) (Allen et al. 2017). Our study showed that combined apatinib and PD-L1 blockade not only caused pruning of the total tumor vessels but also induced intratumoral HEV formation, which trafficked cytotoxic effector $\mathrm{CD} 8^{+} \mathrm{T}$ cells and $\mathrm{CD} 2 \mathrm{O}^{+} \mathrm{B}$ cells into murine MFC gastric tumors. This result prompted us to investigate the mechanism by which combination treatment induces HEV formation. Previous studies have suggested that continuous stimulation of LT $\beta R$ on HEV endothelial cells by CD11 $c^{+}$DC-derived LT $\beta R$ ligands is essential for the induction and maintenance of HEVs (Moussion and Girard 2011; Martinet et al. 2013). We identified that the number of $\mathrm{CD}_{11 \mathrm{C}^{+}} \mathrm{DC}$ s was substantially increased upon combined apatinib and PD-L1 blockade therapy in a mouse model. Clinical data also indicated that the density of mature DC-LAMP ${ }^{+}$DCs was strongly correlated with the density of tumor HEVs in resected gastric adenocarcinoma samples, and the number of DC-LAMP ${ }^{+}$mature DCs and the expression of the LT $\beta R$ and its ligands LT $\beta$ and LIGHT were significantly higher in MSI-H gastric adenocarcinoma tissues than those in MSI-L or MSS GC samples, which may contribute to the increased tumor-associated HEVs formation in MSI-H GC. Moreover, the LT $\beta R$ antagonist greatly inhibited HEV formation and $\mathrm{CD} 8^{+} \mathrm{T}$ cell and $\mathrm{CD} 20^{+} \mathrm{B}$ cell infiltration in MFC tumors undergoing apatinib/anti-PD-L1 therapy. Herein, combined apatinib and anti-PD-L1 therapy triggers HEV formation to promote lymphocyte infiltration through activation of LT $\beta R$ signaling by DCs.

At present, several clinical trials are underway to examine the effects of combined immunotherapy and antiangiogenic regimens in various cancer types (Yi et al. 2019). Clinical studies with antiangiogenic drugs in combination with checkpoint inhibitors have shown a manageable safety profile with favorable antitumor activity in patients with previously treated advanced gastric or gastro-oesophageal junction adenocarcinoma (Herbst et al. 2019). Here, we demonstrated that a combination of apatinib with PD-L1 blockade treatment resulted in more durable and synergistic tumor regression than either single agent 
alone, indicating that combining PD-1/PD-L1 inhibitors and anti-VEGF agents can be an effective treatment strategy to maximize the survival benefit with controllable toxic effects in patients with GC.

In summary, we demonstrated that antiagniogensis drug, apatinib, in combination with PD-L1 blockade synergistically delayed tumor growth and increased survival in an immunocompetent murine model of GC. Such combination reprogrammed the immune microenvironment and promoted antitumor immunity in GC in part through inducing the formation of high endothelial venules (HEVs) through activation of LT $\beta R$ signaling. Our data warrant that apatinib in combination with PD-L1 blockade therapy should be a promising strategy for the treatment of GC in the future clinical practice.

\section{Abbreviations}

GC, gastric cancer; HEVs, high endothelial venules; MSI, Microsatellite instability; MSS, microsatellite stable; PD-L1, programmed death ligand 1; VEGFR2, vascular endothelial growth factor receptor 2; OS, overall survival.

\section{Declarations}

Funding: This work was supported by the following: National Natural Science Foundation of China (81902392); Clinical Research Plan of SHDC (No. SHDC2020CR3033B); Shanghai Committee of Science and Technology (18140900502).

Conflicts of interest: The authors declare that they have no conflict of interest.

Ethics approval: The study was approved by the ethics committee of the Fudan University Shanghai Cancer Center and signed informed consent was obtained from each patient. All animal procedures were performed according to national guidelines and approved by the Institutional Committee of Fudan University for Animal Research.

Consent to participate: Informed consent was obtained from all participants included in this study.

Consent for publication: All authors have consented to publication of the results presented in this manuscript.

Data availability: The datasets supporting the conclusions of this article are included within the article and its additional files.

Authors' contributions: $Y Z$, HRS, FW performed the experiments; $Y Z$ analyzed the data and prepared the manuscript; $Y Z, J P G, Y K H$ and HH provided the samples; HRS, FW, JPG and YKH aided the data analysis. $\mathrm{YZ}$ and $\mathrm{HH}$ wrote the paper; HRS and FW commented on the study and revised the paper; $\mathrm{HH}$ supervised the project; $\mathrm{YZ}$ and $\mathrm{HH}$ designed the research. All authors discussed the results and commented on the manuscript. 
Acknowledgements

This work was supported by the following: National Natural Science Foundation of China (81902392); Clinical Research Plan of SHDC (No. SHDC2020CR3033B); Shanghai Committee of Science and Technology (18140900502).

\section{References}

Allen E, Jabouille A, Rivera LB et al (2017) Combined antiangiogenic and anti-PD-L1 therapy stimulates tumor immunity through HEV formation. Sci Transl Med 9(385). https

://doi.org/10.1126/scitranslmed.aak9679.

Avram G, Sanchez-Sendra B, Martin JM et al (2013) The density and type of MECA-79-positive high endothelial venules correlate with lymphocytic infiltration and tumour regression in primary cutaneous melanoma. Histopathology 63: 852-861. https ://doi.org/10.1111/his.12235.

Bento DC, Jones E, Junaid S et al (2015) High endothelial venules are rare in colorectal cancers but accumulate in extra-tumoral areas with disease progression. Oncoimmunology 4: e974374. https ://doi.org/10.4161/2162402X.2014.974374.

Bray F, Ferlay J, Soerjomataram I et al (2018) Global cancer statistics 2018: GLOBOCAN estimates of incidence and mortality worldwide for 36 cancers in 185 countries. CA Cancer J Clin 68: 394-424. https ://doi.org/10.3322/caac.21492.

Cabrita R, Lauss M, Sanna A et al (2020) Tertiary lymphoid structures improve immunotherapy and survival in melanoma. Nature 577: 561-565. https ://doi.org/10.1038/s41586-019-1914-8.

Chen Y, Ramjiawan RR, Reiberger T et al (2015) CXCR4 inhibition in tumor microenvironment facilitates anti-programmed death receptor-1 immunotherapy in sorafenib-treated hepatocellular carcinoma in mice. Hepatology 61: 1591-1602. https ://doi.org/10.1002/hep.27665.

Cortes-Ciriano I, Lee S, Park WY et al (2017) A molecular portrait of microsatellite instability across multiple cancers. Nat Commun 8: 15180. https ://doi.org/10.1038/ncomms15180.

Eso Y, Shimizu T, Takeda H et al (2020). Microsatellite instability and immune checkpoint inhibitors: toward precision medicine against gastrointestinal and hepatobiliary cancers. J Gastroenterol 55: 15-26. https ://doi.org/10.1007/s00535-019-01620-7

Fridman WH, Zitvogel L, Sautes-Fridman C et al (2017) The immune contexture in cancer prognosis and treatment. Nat Rev Clin Oncol 14: 717-734. https ://doi.org/10.1038/nrclinonc.2017.101.

Geng R, Song L, Li J et al (2018) The safety of apatinib for the treatment of gastric cancer. Expert Opin Drug Saf 17: 1145-1150. https ://doi.org/10.1080/14740338.2018.1535592. 
Gibney GT, Weiner LM, Atkins MB (2016) Predictive biomarkers for checkpoint inhibitor-based immunotherapy. Lancet Oncol 17: e542-e551. https ://doi.org/10.1016/S1470-2045(16)30406-5.

Girard JP, Moussion C, Forster R (2012) HEVs, lymphatics and homeostatic immune cell trafficking in lymph nodes. Nat Rev Immunol 12: 762-773. https ://doi.org/10.1038/nri3298.

Helmink BA, Reddy SM, Gao J et al (2020) B cells and tertiary lymphoid structures promote immunotherapy response. Nature 577: 549-555. https ://doi.org/10.1038/s41586-019-1922-8.

Herbst RS, Arkenau HT, Santana-Davila R et al (2019) Ramucirumab plus pembrolizumab in patients with previously treated advanced non-small-cell lung cancer, gastro-oesophageal cancer, or urothelial carcinomas (JVDF): a multicohort, non-randomised, open-label, phase 1a/b trial. Lancet Oncol 20: 11091123. https ://doi.org/10.1016/S1470-2045(19)30458-9.

Hill DG, Yu L, Gao H et al (2018) Hyperactive gp130/STAT3-driven gastric tumourigenesis promotes submucosal tertiary lymphoid structure development. Int J Cancer 143: 167-178. https ://doi.org/10.1002/ijc.31298.

Huang Y, Yuan J, Righi E et al (2012) Vascular normalizing doses of antiangiogenic treatment reprogram the immunosuppressive tumor microenvironment and enhance immunotherapy. Proc Natl Acad Sci U S A 109: 17561-17566. https ://doi.org/10.1073/pnas.1215397109.

Jayson GC, Kerbel R, Ellis LM et al (2016) Antiangiogenic therapy in oncology: current status and future directions. Lancet 388: 518-529. https ://doi.org/10.1016/S0140-6736(15)01088-0.

Kandalaft LE, Motz GT, Busch J et al (2011) Angiogenesis and the tumor vasculature as antitumor immune modulators: the role of vascular endothelial growth factor and endothelin. Curr Top Microbiol Immunol 344: 129-148. https ://doi.org/10.1007/82_2010_95.

Le DT, Durham JN, Smith KN et al (2017) Mismatch repair deficiency predicts response of solid tumors to PD-1 blockade. Science 357: 409-413. https ://doi.org/10.1126/science.aan6733.

Lee SJ, Jang BC, Lee SW et al (2006) Interferon regulatory factor-1 is prerequisite to the constitutive expression and IFN-gamma-induced upregulation of B7-H1 (CD274). FEBS Lett 580: 755-762. https ://doi.org/10.1016/j.febslet.2005.12.093.

Liu XD, Hoang A, Zhou L et al (2015) Resistance to Antiangiogenic Therapy Is Associated with an Immunosuppressive Tumor Microenvironment in Metastatic Renal Cell Carcinoma. Cancer Immunol Res 3: 1017-1029. https ://doi.org/10.1158/2326-6066.CIR-14-0244.

Mahmoud SM, Paish EC, Powe DG et al (2011) Tumor-infiltrating CD8+ lymphocytes predict clinical outcome in breast cancer. J Clin Oncol 29: 1949-1955. https ://doi.org/10.1200/JC0.2010.30.5037. 
Martinet L, Filleron T, Le Guellec S et al (2013) High endothelial venule blood vessels for tumor-infiltrating lymphocytes are associated with lymphotoxin beta-producing dendritic cells in human breast cancer. J Immunol 191: 2001-2008. https ://doi.org/10.4049/jimmunol.1300872.

Martinet L, Garrido I, Filleron T et al (2011) Human solid tumors contain high endothelial venules: association with $\mathrm{T}$ - and B-lymphocyte infiltration and favorable prognosis in breast cancer. Cancer Res 71: 5678-5687. https ://doi.org/10.1158/0008-5472.CAN-11-0431.

Meder L, Schuldt P, Thelen M et al (2018) Combined VEGF and PD-L1 Blockade Displays Synergistic Treatment Effects in an Autochthonous Mouse Model of Small Cell Lung Cancer. Cancer Res 78: 42704281. https ://doi.org/10.1158/0008-5472.CAN-17-2176.

Moussion C, Girard JP (2011) Dendritic cells control lymphocyte entry to lymph nodes through high endothelial venules. Nature 479: 542-546. https ://doi.org/10.1038/nature10540.

Noman MZ, Desantis G, Janji B et al (2014) PD-L1 is a novel direct target of HIF-1alpha, and its blockade under hypoxia enhanced MDSC-mediated T cell activation. J Exp Med 211: 781-790. https ://doi.org/10.1084/jem.20131916.

Petitprez F, de Reynies A, Keung EZ et al (2020) B cells are associated with survival and immunotherapy response in sarcoma. Nature 577: 556-560. https ://doi.org/10.1038/s41586-019-1906-8.

Pfuderer PL, Ballhausen A, Seidler F et al (2019) High endothelial venules are associated with microsatellite instability, hereditary background and immune evasion in colorectal cancer. $\mathrm{Br} \mathrm{J}$ Cancer 121: 395-404. https ://doi.org/10.1038/s41416-019-0514-6.

Pitt JM, Vetizou M, Daillere R et al (2016) Resistance Mechanisms to Immune-Checkpoint Blockade in Cancer: Tumor-Intrinsic and -Extrinsic Factors. Immunity 44: 1255-1269. https ://doi.org/10.1016/j.immuni.2016.06.001.

Ramjiawan RR, Griffioen AW, Duda DG (2017) Anti-angiogenesis for cancer revisited: Is there a role for combinations with immunotherapy? Angiogenesis 20: 185-204. https ://doi.org/10.1007/s10456-0179552-y.

Rosen SD (2004) Ligands for L-selectin: homing, inflammation, and beyond. Annu Rev Immunol 22: $129-$ 156. https ://doi.org/10.1146/annurev.immunol.21.090501.080131.

Russo AE, Strong VE (2019) Gastric Cancer Etiology and Management in Asia and the West. Annu Rev Med 70: 353-367. https ://doi.org/10.1146/annurev-med-081117-043436.

Samarajiwa SA, Forster S, Auchettl K et al (2009) INTERFEROME: the database of interferon regulated genes. Nucleic Acids Res 37: D852-D857. https ://doi.org/10.1093/nar/gkn732. 
Sato J, Kitano S, Motoi N et al (2020) CD20(+) tumor-infiltrating immune cells and CD204(+) M2 macrophages are associated with prognosis in thymic carcinoma. Cancer Sci 111: 1921-1932. https ://doi.org/10.1111/cas.14409.

Sautes-Fridman C, Petitprez F, Calderaro J et al (2019) Tertiary lymphoid structures in the era of cancer immunotherapy. Nat Rev Cancer 19: 307-325. https ://doi.org/10.1038/s41568-019-0144-6.

Schmittnaegel M, Rigamonti N, Kadioglu E et al (2017) Dual angiopoietin-2 and VEGFA inhibition elicits antitumor immunity that is enhanced by PD-1 checkpoint blockade. Sci Transl Med 9(385). https ://doi.org/10.1126/scitranslmed.aak9670.

Shigeta K, Datta M, Hato T et al (2020) Dual Programmed Death Receptor-1 and Vascular Endothelial Growth Factor Receptor-2 Blockade Promotes Vascular Normalization and Enhances Antitumor Immune Responses in Hepatocellular Carcinoma. Hepatology 71: 1247-1261. https ://doi.org/10.1002/hep.30889.

von Andrian UH, Mempel TR (2003) Homing and cellular traffic in lymph nodes. Nat Rev Immunol 3: 867878. https ://doi.org/10.1038/nri1222.

Wculek SK, Cueto FJ, Mujal AM et al (2020). Dendritic cells in cancer immunology and immunotherapy. Nat Rev Immunol 20: 7-24. https ://doi.org/10.1038/s41577-019-0210-z

Wirsing AM, Ervik IK, Seppola M et al (2018) Presence of high-endothelial venules correlates with a favorable immune microenvironment in oral squamous cell carcinoma. Mod Pathol 31: 910-922. https ://doi.org/10.1038/s41379-018-0019-5.

Yi M, Jiao D, Qin S et al (2019) Synergistic effect of immune checkpoint blockade and anti-angiogenesis in cancer treatment. Mol Cancer 18: 60. https ://doi.org/10.1186/s12943-019-0974-6.

\section{Figures}


A

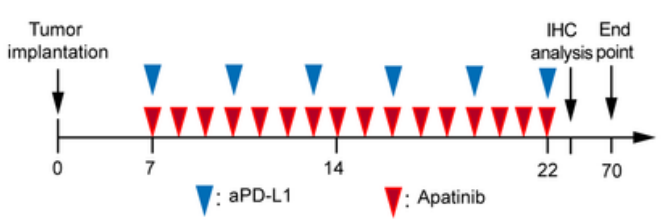

D

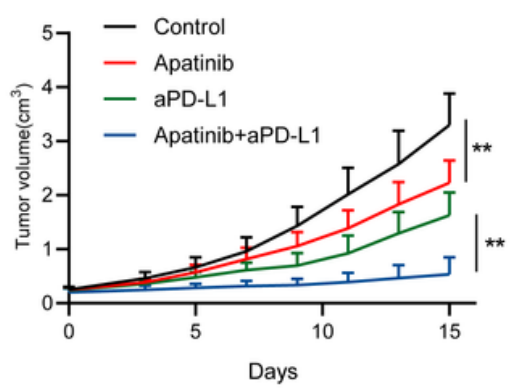

B

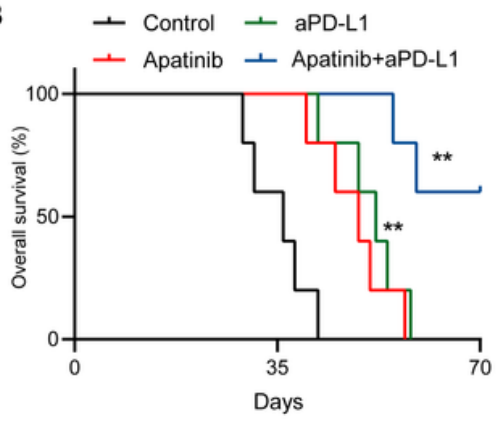

E

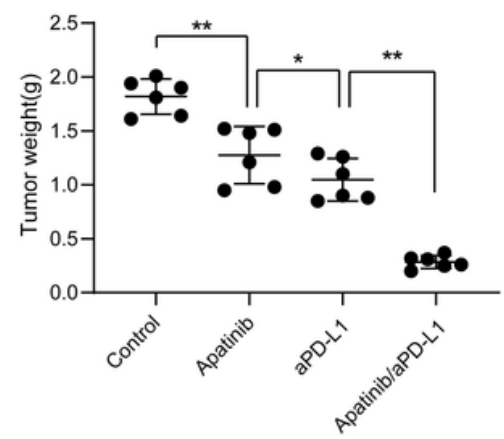

C $\rightarrow$ Expected additive survival rate (Apatinib/aPD-L1)

- Observed survival rate(Apatinib/aPD-L1)

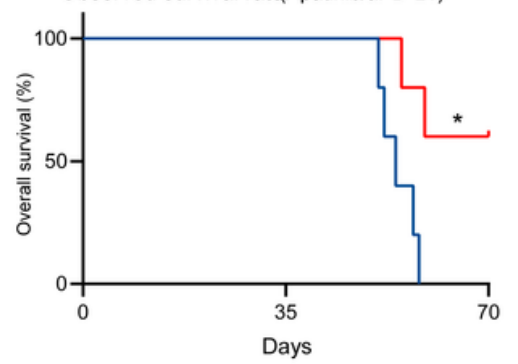

$\mathbf{F}$

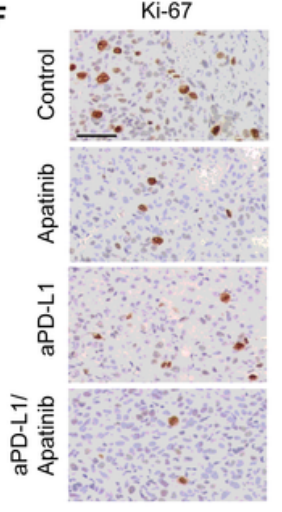

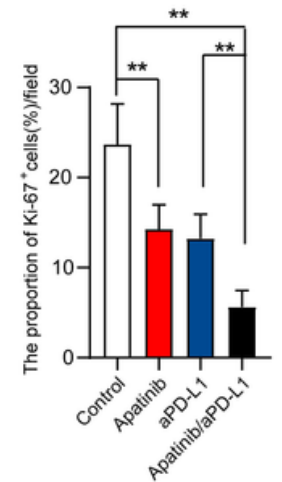

\section{Figure 1}

Combined apatinib and PD-L1 blockade therapy synergistically inhibited tumor growth and improved OS in a mouse model. a Summary of drug intervention protocol. b Survival curves of MFC-bearing mice treated with solvent control $(n=5)$, apatinib monotherapy $(n=5)$, anti-PD-L1 monotherapy (aPD-L1, $n=5)$ and combined apatinib/aPD-L1 therapy $(n=5)$. c The predicted additive OS curves were calculated as described in supplementary methods and compared with the curves of observed combination therapy using Prism Mantel Cox test. d-e Tumor growth curves and tumor weights of MFC-bearing mice treated with solvent control $(n=6)$, apatinib monotherapy $(n=6)$, anti-PD-L1 monotherapy (aPD-L1, $n=6)$ and combined apatinib/aPD-L1 therapy $(n=6) . f$ Immunohistochemical staining of tumor proliferative marker ki-67 in harvested tumors. Representative immunohistochemical images were shown. Data are shown as the mean \pm SD of three independent experiments. *: $P<0.05$; $* *$ : $<<0.01$; NS: No Significance. Scale bars: $50 \mu \mathrm{m}$. 
A

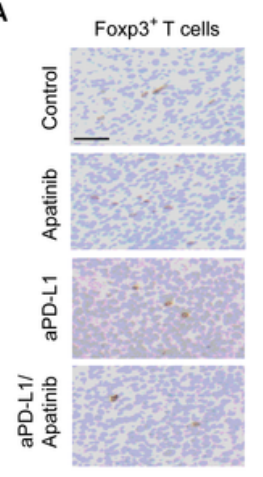

B

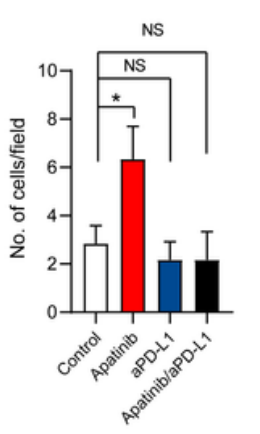

B $\quad \operatorname{CD8}^{+} \mathrm{T}$ cells

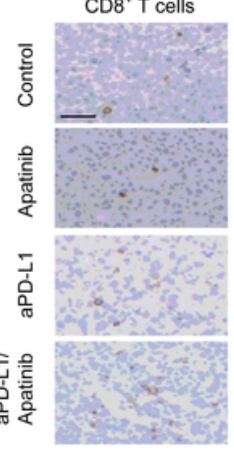

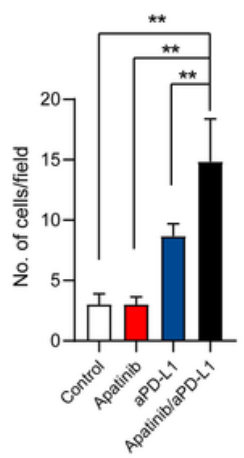

C
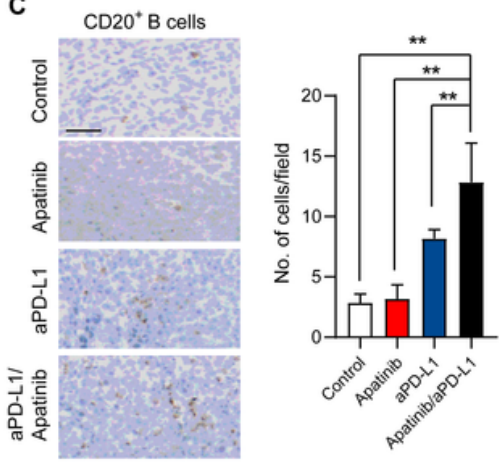

$\mathbf{F}$
D

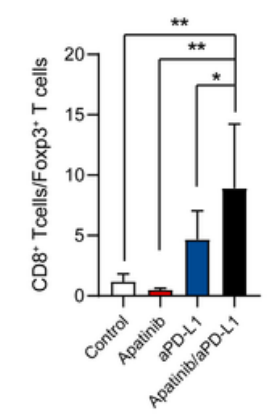

Th1/Th2

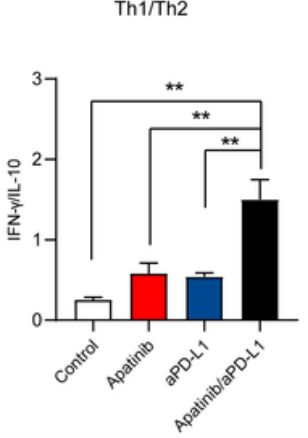

E

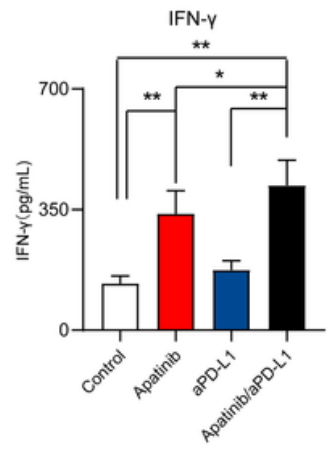

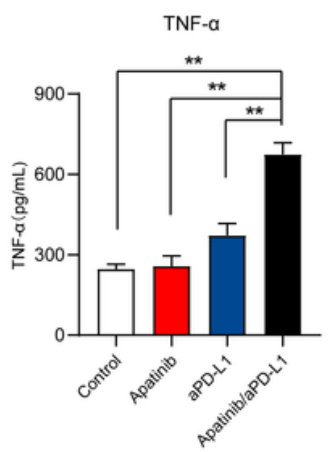

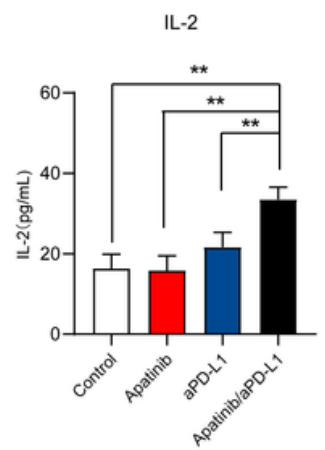

IL-10

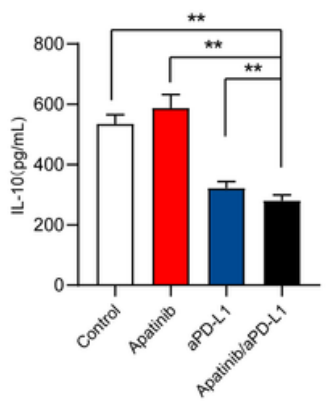

Figure 2

The effect of apatinib in combination with PD-L1 blockade on immune environment in a GC mouse model. a-c Immunohistochemistry was performed to detect the changes in tumor infiltrating FoxP3+ Treg cells (a), CD8+ CTLs (b) and CD20+B cells (c) in MFC tumors treated with solvent control, apatinib monotherapy, anti-PD-L1 monotherapy and combined apatinib/aPD-L1 therapy. Representative immunohistochemical images were shown. $d$ Changes in ratios of CD8+ CTLs to FoxP3+ Tregs in MFC tumors treated with solvent control, apatinib monotherapy, anti-PD-L1 monotherapy and combined apatinib/aPD-L1 therapy. e Changes in the levels of IFN- $\gamma$, TNFa and IL-2 in plasma of MFC-bearing mice treated with solvent control, apatinib monotherapy, anti-PD-L1 monotherapy and combined apatinib/aPDL1 therapy. $f$ Changes in the ratios of the Th1/Th2 cytokine (IFN-y/IL-10) in plasma of MFC-bearing mice treated with solvent control, apatinib monotherapy, anti-PD-L1 monotherapy and combined apatinib/aPDL1 therapy. Data are shown as the mean \pm SD of three independent experiments. *: $P<0.05$; $\star *: P<0.01$; NS: No Significance. Scale bars: $50 \mu \mathrm{m}$. 
A

PD-L1
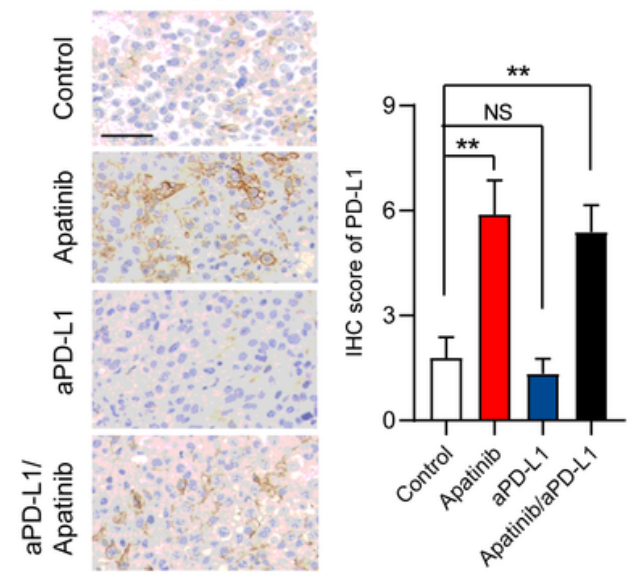

B
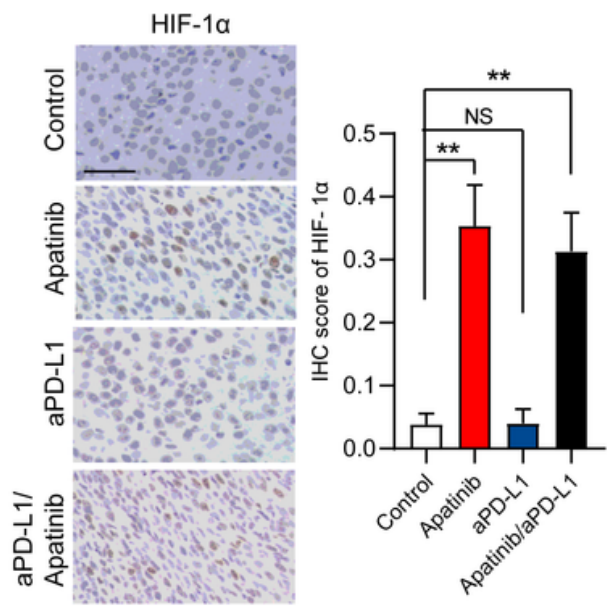

C

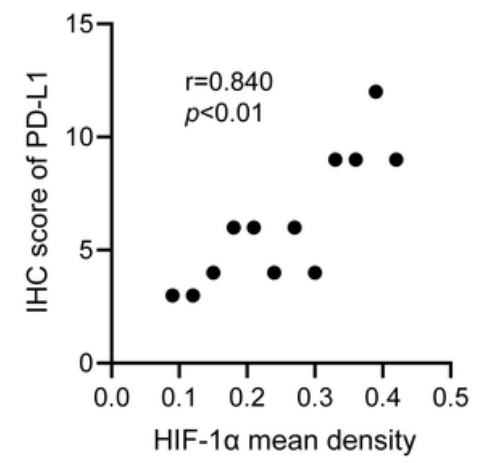

D

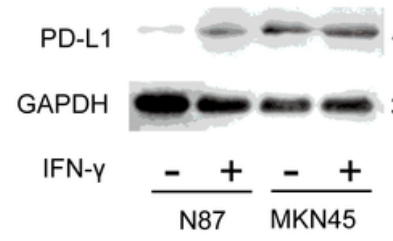

$45 \mathrm{KD}$

$35 \mathrm{KD}$

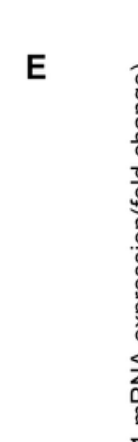

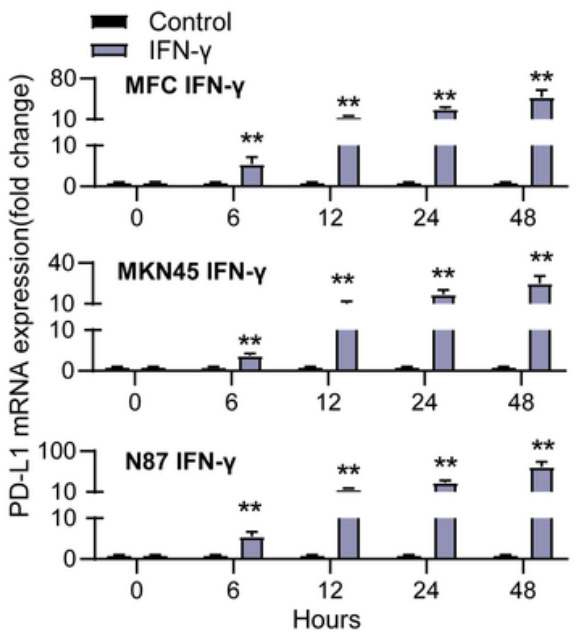

F

口 Control घ Apatinib - aPD-L1 - Apatinib/aPD-L1

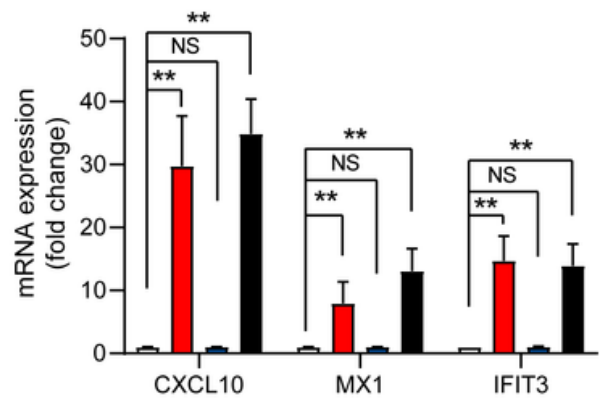

\section{Figure 3}

Hypoxia and IFN-y expression contributed to the upregulation of PD-L1 induced by apatinib treatment. a Immunohistochemistry was performed to detect the PD-L1 expression in MFC tumors treated with solvent control, apatinib monotherapy, anti-PD-L1 monotherapy and combined apatinib/aPD-L1 therapy. Representative immunohistochemical images were shown. $b$ Immunohistochemistry was performed to detect the HIF-1a expression in MFC tumors treated with solvent control, apatinib monotherapy, anti-PDL1 monotherapy and combined apatinib/aPD-L1 therapy. Representative immunohistochemical images were shown. c Scatter plot diagram showed significantly positive correlation between HIF-1a and PD-L1 expression in MFC tumor tissues treated with apatinib. $d$ Western blot analysis of PD-L1 in MKN45 and N87 GC cells treated for 48 hours with recombinant human IFN- $\gamma(10 \mathrm{ng} / \mathrm{ml})$. e qRT-PCR was performed to analyze the expression of PD-L1 in MKN45 and N87 human and MFC mouse GC cell lines treated for 48 hours with recombinant mouse or human IFN- $\mathrm{\gamma}(10 \mathrm{ng} / \mathrm{ml})$ at indicated times. $\mathrm{f}$ qRT-PCR was performed to analyze the expression of IFNy-mediated genes CXCL10, MX1, and IFIT3 in cells isolated from MFC tumors treated with solvent control, apatinib monotherapy, anti-PD-L1 monotherapy and combined apatinib/aPD-L1 therapy. Data are shown as the mean \pm SD of three independent experiments. *: $P<0.05$; **: P<0.01; NS: No Significance. Scale bars: $50 \mu \mathrm{m}$. 
A

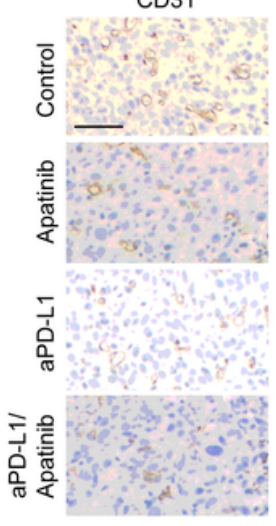

B

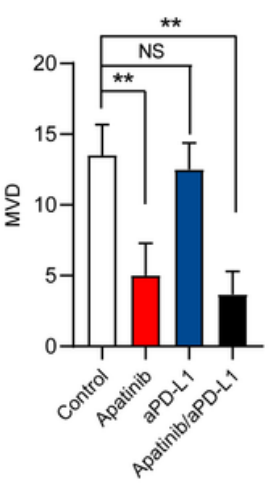

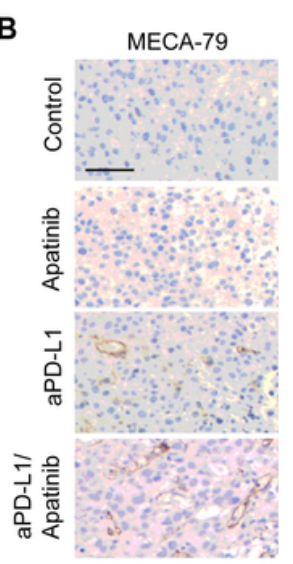

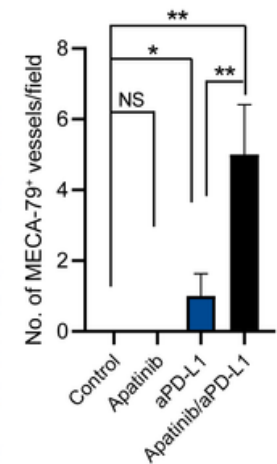

C

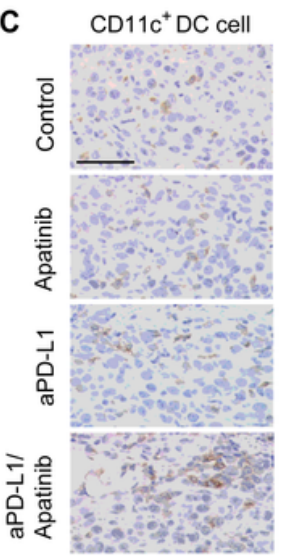

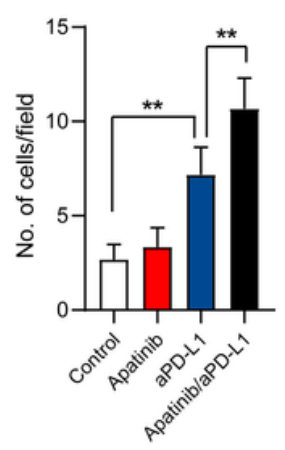

D

E
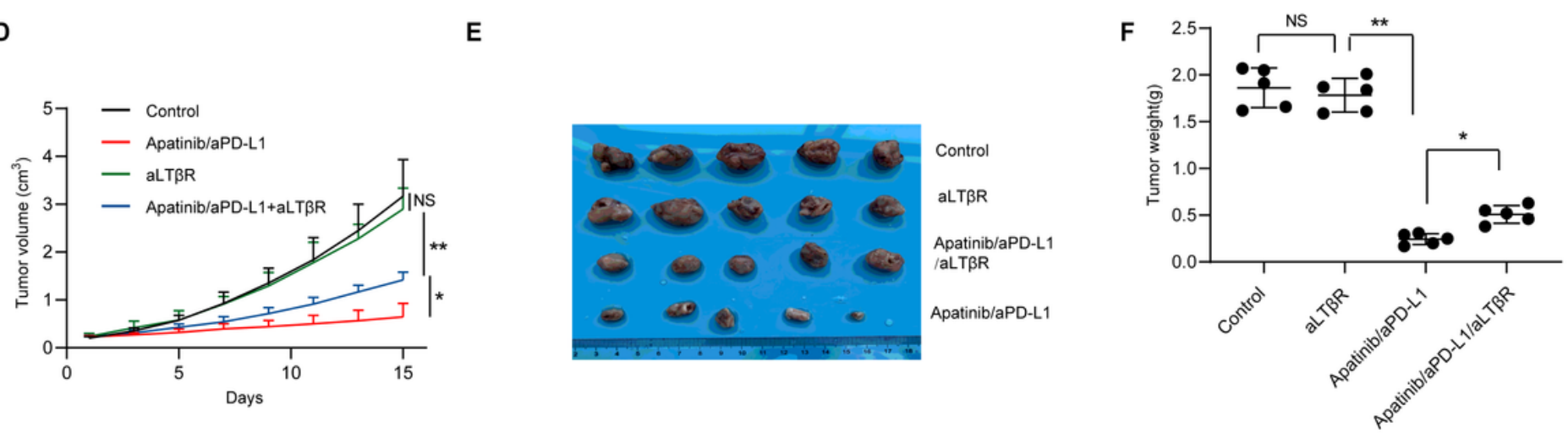

G
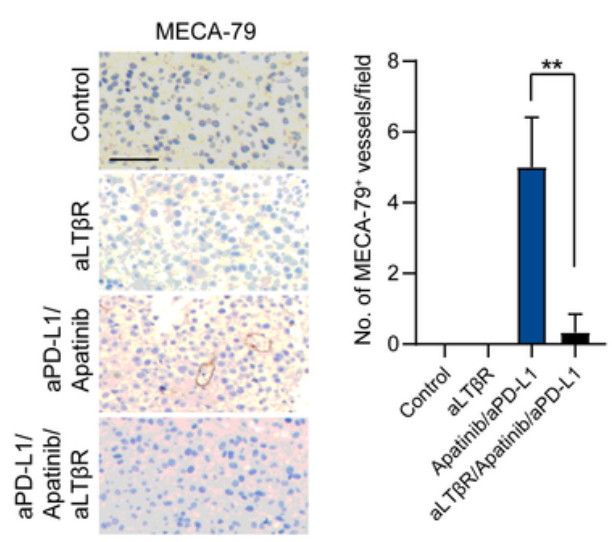

H

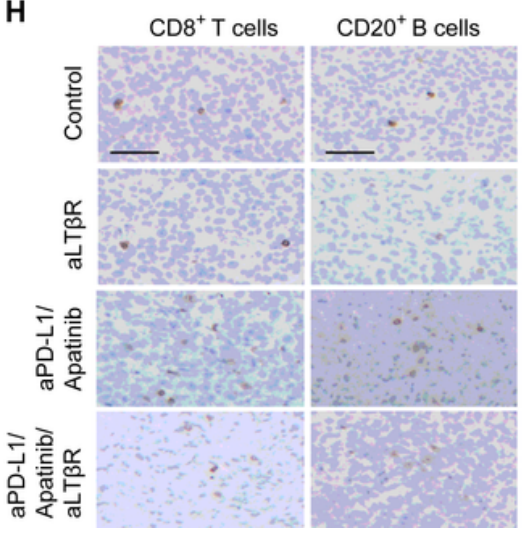

$=\mathrm{CD}^{+} \mathrm{T}$ cells

- $\mathrm{CD}^{2} 0^{+} \mathrm{B}$ cells

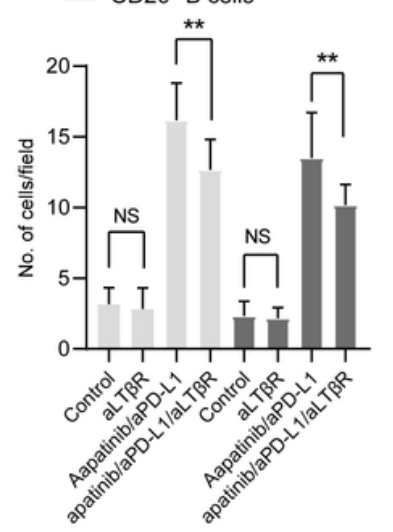

Figure 4

LT $\beta$ R signaling activation mediated by dendritic cells contributed to the HEVs formation induced by dual apatinib/ PD-L1 blockade therapy. a-c Immunohistochemistry was performed to detect the MVD-CD31(a), MECA-79+ HEVs(b) and CD11C+ DCs(c) in MFC tumors treated with solvent control, apatinib monotherapy, anti-PD-L1 monotherapy and combined apatinib/aPD-L1 therapy. Representative immunohistochemical images were shown. $d$-f Tumor growth curves $(d)$, representative images of subcutaneous MFC mouse tumors (e) and tumor weights ( $f$ ) of MFC-bearing mice treated with solvent control $(n=5)$, LT $\beta R$ signaling antagonistic antibody $(n=5)$, combined apatinib/aPD-L1 therapy $(n=5)$ and combined apatinib/aPD-L1/ LT $\beta R$ signaling antagonistic antibody therapy $(n=5) . g-h$ Immunohistochemistry was performed to detect the MECA-79+ HEVs (g), CD8+ CTLs and CD20+B cells 
(h) in MFC tumors treated with solvent control, LT $\beta R$ signaling antagonistic antibody(aLT $\beta R$ ), combined apatinib/aPD-L1 therapy and combined apatinib/aPD-L1/aLT $\beta R$. Representative immunohistochemical images were shown. Data are shown as the mean \pm SD of three independent experiments. *: $P<0.05$; **: P<0.01; NS: No Significance. Scale bars: $50 \mu \mathrm{m}$.
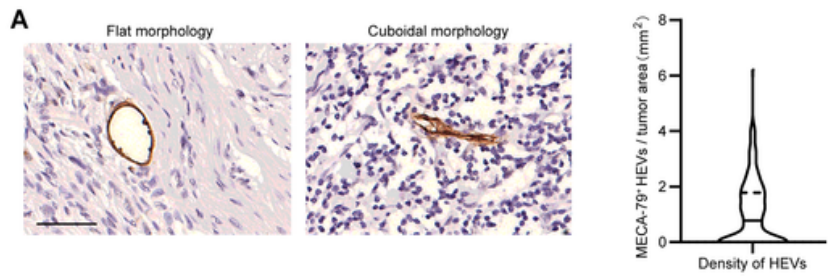

C

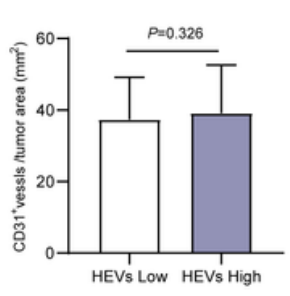

D
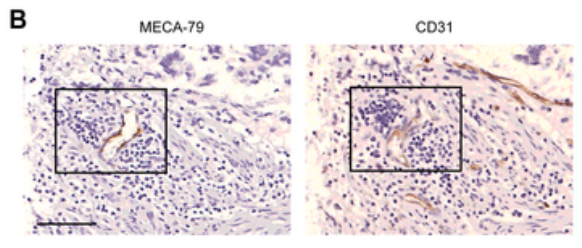

E

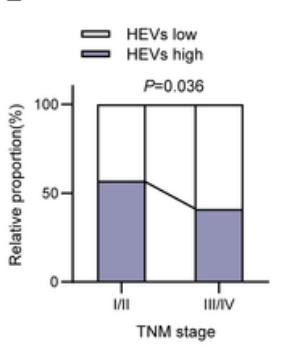

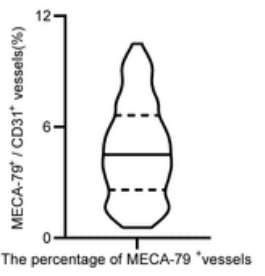

$\mathbf{F}$

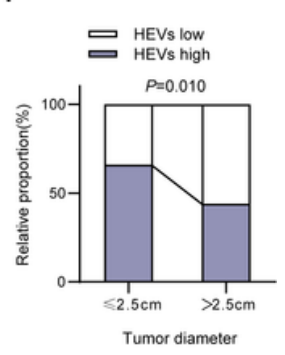

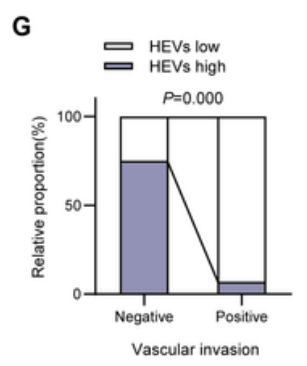

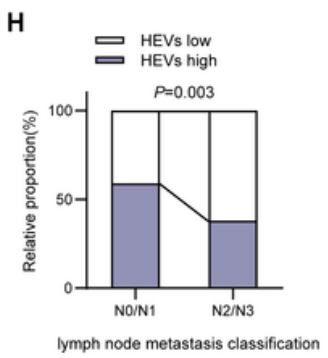

I
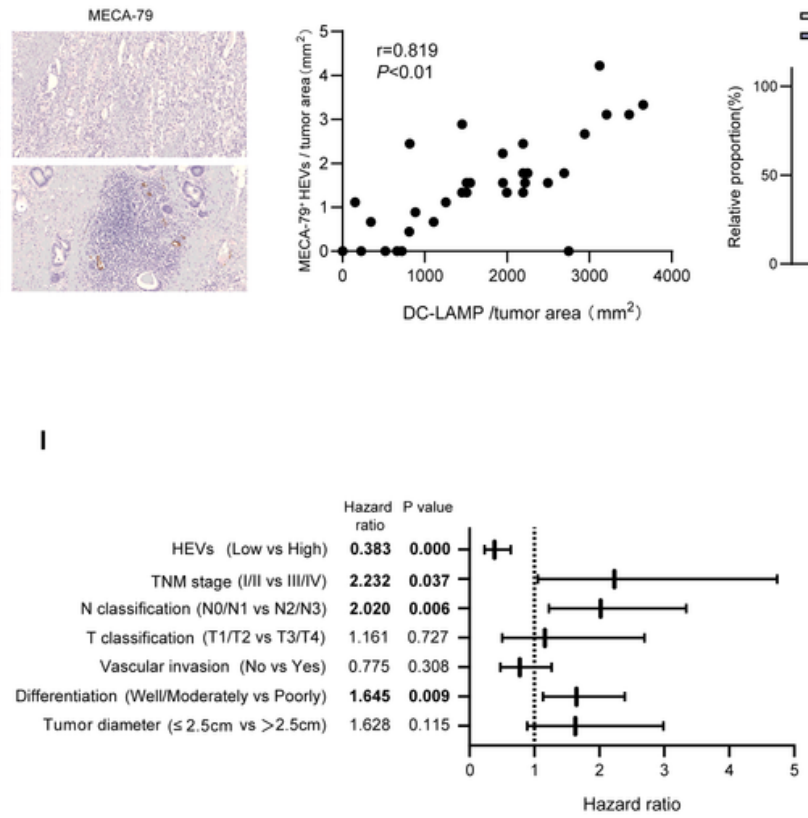

$J$

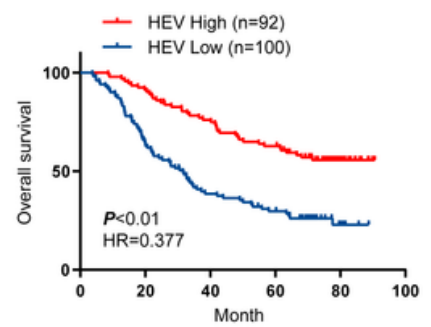

Figure 5

Expression and prognostic value of HEVs in GC patients. a Immunohistochemistry was performed to detect the phenotypic characterization of tumor MECA-79+ HEVs in gastric adenocarcinoma patients. Representative immunohistochemical images were shown. Scale bars: $50 \mu \mathrm{m}$ b-c Consecutive GC sections were stained by immunohistochemistry with anti-MECA-79 and CD31 antibodies (b). The density of CD31+ vessels was compared between HEV-high ( $\geq 5$ per view of field, $n=92$ ) and HEV-low ( $₫ 5$ per view of field, $n=100$ ) gastric adenocarcinoma(c). Representative immunohistochemical images were shown. Scale bars: $50 \mu \mathrm{m}$. d Consecutive gastric adenocarcinoma sections were stained by immunohistochemistry with anti-MECA-79 and DC-LAMP(LAMP3) antibodies. Scatter plot diagram showed significantly positive correlation between MECA-79+ HEVs and mature DCs (DC-LAMP) in GC samples. Scale bars: $250 \mu \mathrm{m}$. e-h High density of HEVs negatively correlated with TNM (tumor node metastasis) stages(e), tumor diameter(f), vascular invasion( $(\mathrm{g})$ and lymph node metastasis(h) in gastric adenocarcinoma patients. i Kaplan-Meier curves for overall survival after GC resection according to MECA-79+ HEVs densities in human gastric adenocarcinoma tissues $(n=192)$. HEV-high: ( $\geq 5$ per view of field, $n=92$ ); HEV-low: ( $₫ 5$ per view of field, $n=100$ ). j Forest plots showing HR of OS for high-risk patients in the indicated clinical subgroups of gastric adenocarcinoma patients. 
A

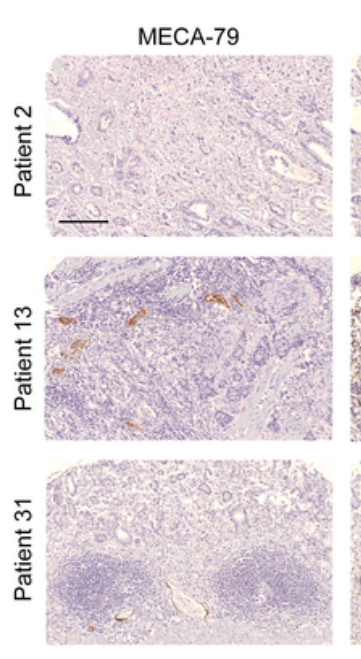

$\mathrm{CD} 3$
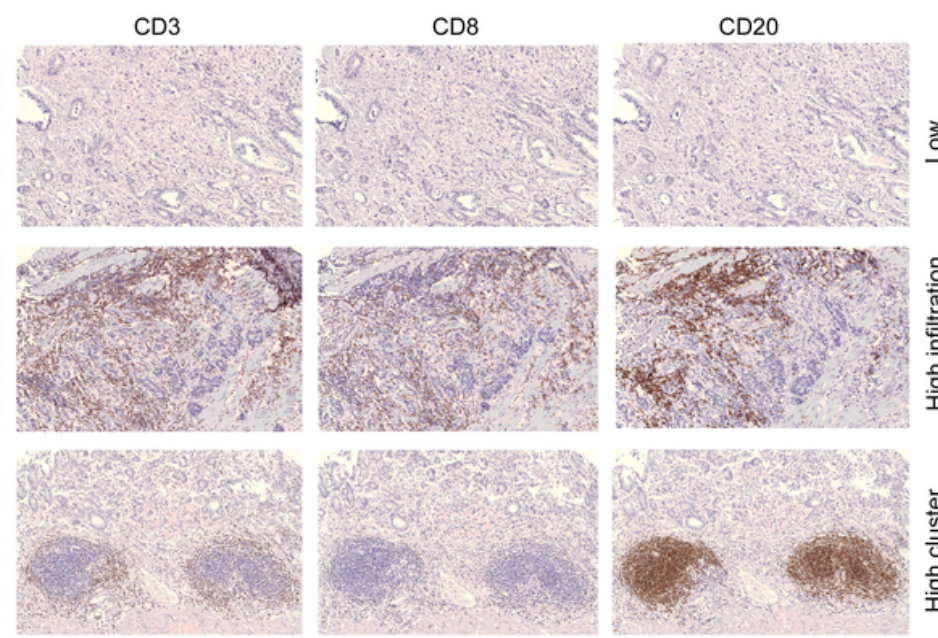

క̧

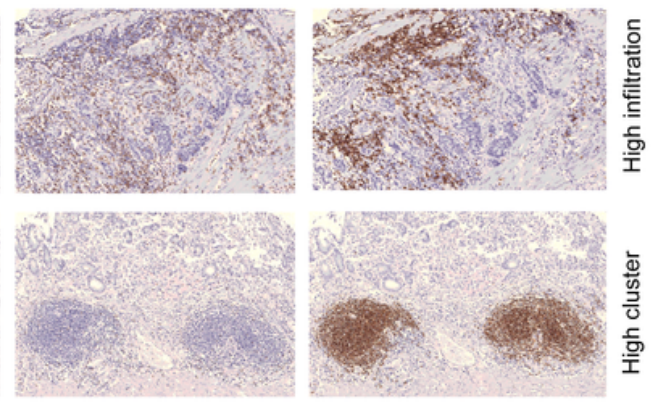

B
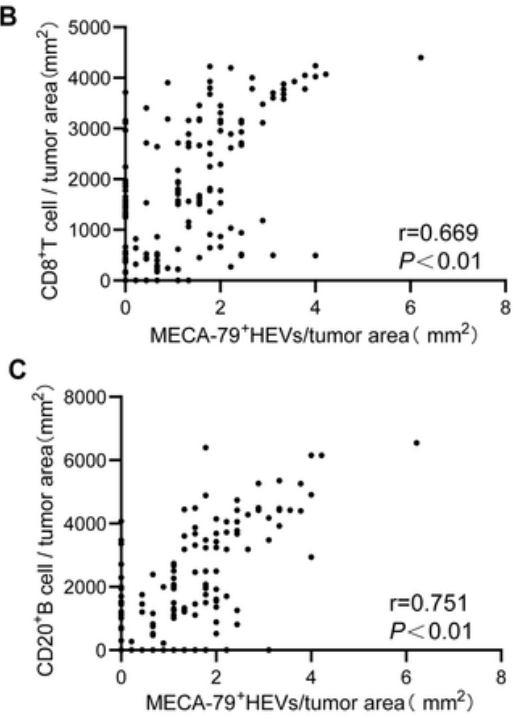

D

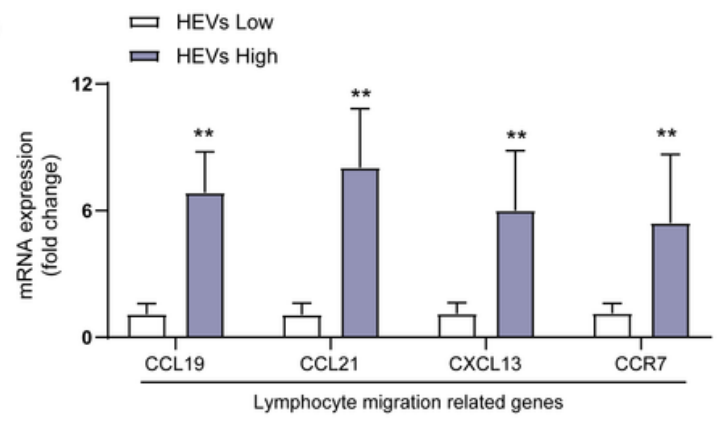

G

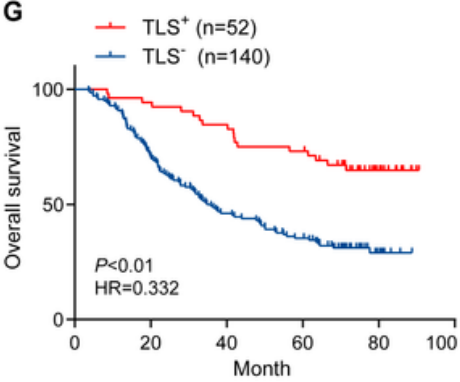

H $-\mathrm{CD}^{+} \mathrm{T}$ cell High ( $\mathrm{n}=96$ )

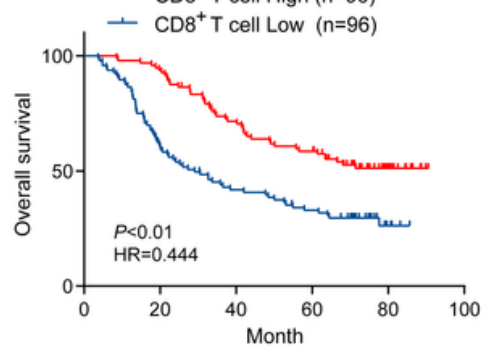

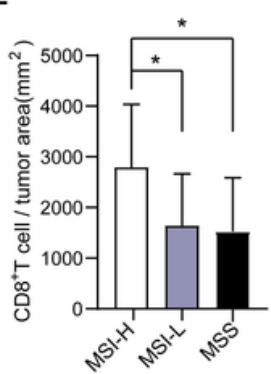

$\mathbf{F}$

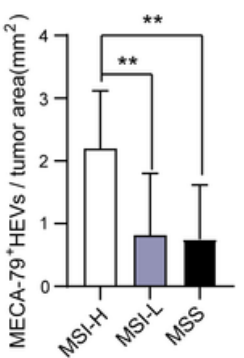

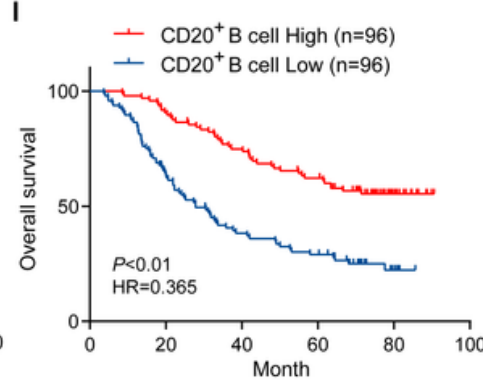

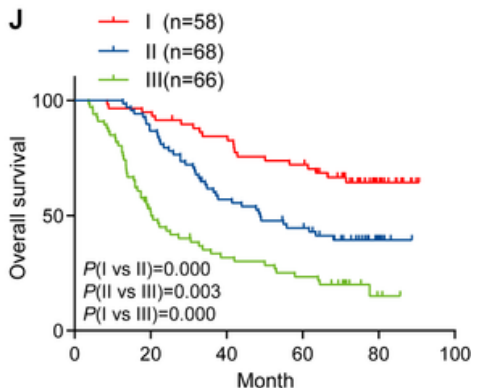

Figure 6

HEVs predicts lymphocyte infiltration into the tumor in GC patients a Representative consecutive GC sections showing the expression of MECA-79+, CD3+ T cells, CD8+ T cells and CD20+ B cells in gastric adenocarcinoma samples. b-c Scatter plot diagram showed significantly positive correlation between MECA-79+ HEVs and CD8+ T cells as well as CD20+ B cells in gastric adenocarcinoma samples. $d$ Expression of genes related to lymphocyte migration determined in 18 gastric adenocarcinoma samples according to the density of tumor HEVs. HEV-high: $(n=8)$; HEV-low: $(n=10)$. e The intratumoral CD8+ T cells and CD20+ B cells in MSI-H(n=10), MSI-L(n=11) and MSS( $n=12)$ gastric adenocarcinoma samples detected by immunohistochemical staining. $f$ HEV density in MSI-H( $n=10), M S I-L(n=11)$ and MSS( $n=12)$ gastric adenocarcinoma samples detected by immunohistochemical staining. g Kaplan-Meier survival curve of gastric adenocarcinoma patients with positive $(n=52)$ and negative $(n=140)$ TLS. h-i KaplanMeier survival curve of gastric adenocarcinoma patients with lower ( $\$ 5623$ per view of field, $n=96)$ and 
higher $(\geq 5623$ per view of field, $n=96)$ CD8 $+T$ cells counts, or with lower ( $(4564$ per view of field, $n=96$ ) and higher ( $\geq 4564$ per view of field, $n=96$ ) CD20+B cells counts. $j$ The combined quantity of intratumoral CD8+ $T$ cells, CD20+ B cells, and MECA-79+ HEVs predicted the prognosis of gastric adenocarcinoma patients. I:CD8+ T cells high/ CD20+ B cells high / MECA-79+ HEVs high ( $n=58) ;$ Ill:CD8+ T cells low/ CD20+ B cells low/ MECA-79+ HEVs low $(n=66)$; Il: other $(n=68)$. Data are shown as the mean \pm SD of three independent experiments. *: $\mathrm{P}<0.05$; $* *$ : $\mathrm{P}<0.01$; NS: No Significance. Scale bars: $250 \mu \mathrm{m}$.

\section{Supplementary Files}

This is a list of supplementary files associated with this preprint. Click to download.

- R1Additionalfile1SupplementaryFiguresJOCRD2100011.docx

- R1Additionalfile2SupplementaryTablesJOCRD2100011.docx

- R1Additionalfile3SupplementaryMethodsJOCRD2100011.docx 